EGU21-6223

https://doi.org/10.5194/egusphere-egu21-6223

EGU General Assembly 2021

(c) Author(s) 2021. This work is distributed under

the Creative Commons Attribution 4.0 License.

\title{
Role of the heliospheric current sheet in high energy proton transport through modelling of historic GLE events
}

Charlotte Waterfall and Silvia Dalla

University of Central Lancashire, Natural Sciences, United Kingdom of Great Britain - England, Scotland, Wales (cwaterfall@uclan.ac.uk)

The influence of the heliospheric current sheet (HCS) on the propagation of high energy solar protons is explored using 3D test particle modelling. The test particle model, which includes drift effects, is used to simulate specific past ground level enhancement (GLE) events which cover a range of HCS configurations. For example, the effects of a source location close to and far from the HCS for events both poorly and well-connected to Earth are examined. Similarly, the effect of the Earth's location relative to the HCS is explored. The modelling is performed for high energy (300-1200 MeV) protons to represent the energetic conditions under which GLEs occur. The derived intensity profiles at $1 \mathrm{AU}$ are compared to observations from HEPAD onboard GOES, as well as STEREO (at locations away from Earth) and neutron monitor data. 